ÉGYPTE

monde arabe

\section{Égypte/Monde arabe}

4 | 1990

Démocratie et démocratisation dans le monde arabe

\title{
Les partis (pris) démocratiques. Perceptions occidentales de la démocratisation dans le monde arabe
}

Jean-Claude Vatin

\section{(2) OpenEdition \\ Journals}

Édition électronique

URL : https://journals.openedition.org/ema/338

DOI : 10.4000/ema.338

ISSN : 2090-7273

Éditeur

CEDEJ - Centre d'études et de documentation économiques juridiques et sociales

Édition imprimée

Date de publication : 31 décembre 1990

ISSN : 1110-5097

Référence électronique

Jean-Claude Vatin, « Les partis (pris) démocratiques. Perceptions occidentales de la démocratisation dans le monde arabe », Égypte/Monde arabe [En ligne], 4 | 1990, mis en ligne le 08 juillet 2008, consulté le 07 juillet 2022. URL : http://journals.openedition.org/ema/338 ; DOI : https://doi.org/10.4000/ema. 338

Ce document a été généré automatiquement le 7 juillet 2022.

Tous droits réservés 


\title{
Les partis (pris) démocratiques. Perceptions occidentales de la démocratisation dans le monde arabe
}

\author{
Jean-Claude Vatin
}

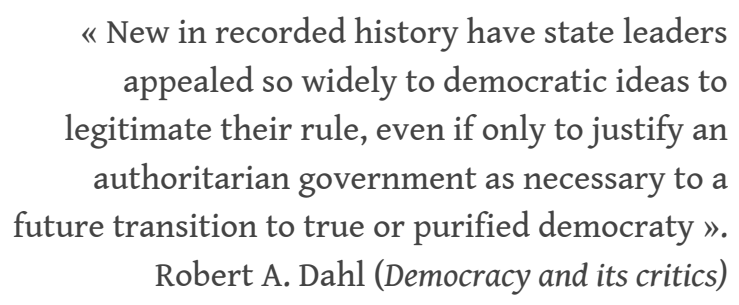

1 Dans un article publié en $1984^{1}$, un des principaux théoriciens du développement politique, Samuel Huntington, l'auteur de Political order in changing societies (1967), se demandait - et c'était le titre - «Will more countries become democratic? " (Political science quarterly $9-\mathrm{n}^{\circ} 2, \mathrm{p} .193-218$ ). Et la même question se retrouve implicite dans les chapitres rassemblés par Guillermo O'Donnell, Philippe C. Schmitter et Laurence Whitehead: Transitions from authoritarian rule. Prospects for democracy (1986). Il me semble qu'on tient là une des reconversions ou glissements dont la politotogie contemporaine a le secret.

2 Après les analyses institutionnelles en honneur dans l'entre-deux guerres mondiales, puis celles centrées sur la culture politique, la modernisation, notamment, en fonction de critères économiques, qui ont prévalu jusque dans les années 1970, on en est revenu, durant la dernière décennie, à des recherches moins théorisées si j'ose dire, les unes plus liées aux transformations historiques des sociétés arabes, les autres relevant d'approches culturelles. Avec un retour à l'État, à travers la dynamique créée par les relations contrariées mais imposées entre société civile et appareils de pouvoir. Et, par une série de détours, nous voici dans la logique du vieux débat cher aux philosophes européens du $\mathrm{XVIII}^{\mathrm{e}}$ siècle, au rapport individu-collectivité politique, citoyen-État. En 
réalité, sous l'impact des mouvements islamistes (le «réveil» de l'islam), les paradigmes occidentaux (appelons-les de substitution) ont perdu de leur universalité. Et même si les modèles orientaux continuent à être récusés, les réexamens en cours démontrent que les perceptions sont en train d'évoluer.

Le réveil par l'islam

3 Sans vouloir être provocateur, j'avancerais volontiers que les mutations récentes que l'on vient d'évoquer et qui conduisent à un certain désenchantement pourraient tenir, pour partie au moins, a ce que la critique occidentale a nommé un peu abusivement le " retour » de l'islam, la "remontée » du religieux, ce à quoi on se réfère de façon tout aussi hasardeuse sous l'appellation d'islamisme. Au fond, la politologie occidentale doit beaucoup à l'imam Khomeiny et au renversement de la dynastie iranienne provoqué et mené par des leaders religieux, suivi de l'établissement d'un pouvoir qualifié de "mollarchie» par quelques amateurs sans complexe, ou de semi-théocratie par d'autres plus sensibles au respect des taxinomies traditionnelles.

4 A partir de ce moment, les théoriciens politiques - ceux qui se préoccupaient plus d'évaluer les forces sociales en présence, les capacités d'adaptation, les potentiels économiques au sein d'une communauté politique, d'un État national, par exemple, en fonction de paramètres et critères applicables à l'ensemble des sociétés existantes d'un certain type ou genre (le Tiers-monde, les pays en voie de développement...) - ont dû soit reculer et céder la place aux spécialistes des aires culturelles musulmanes soit passer contrat avec eux ou utiliser leurs travaux. Dès 1980 et pour plusieurs années, se sont trouvées relancées des études où était réintroduite en force (et non sans système donc artificialité), l'islamologie largement délaissée jusqu'ici, pour expliquer les phénomènes politiques observés dans le monde arabe.

5 La «dimension" musulmane, pour qui étudiait l'Égypte de Nasser et de Sadate (pensons à P. J Vatikiotis : Nasser and his generation, 1978, à John Waterbury : Egypt of Nasser and Sadat, 1983, ou à Raymond A. Hinnebush, Egyptian politics under Sadat. The post populist development of an authoritarian modernizing State, 1985), l'Algérie de Boumediene (cf. Jean Leca et Jean-Claude VATIN : L'Algérie, Institutions et régime, 1975) ou le parti Ba'th tel qu'installé en Syrie et en Irak (référons-nous à John Devlin : The Bath Party, 1976), n'était prise en compte qu'à l'arrière-plan, à la limite comme un instrument de manipulation politique dont usaient leaders en place et contre-leaders potentiels. Il n'était donc guère besoin de faire entrer l'islam en ligne de compte sinon comme une donnée (input) supplémentaire nommée : confréries, Frères musulmans, communauté chiite, néo-réformisme, islam radical et jama'at par exemple.

6 Soudain, ce qui était advenu à Téhéran en 1979 puis au Caire à l'occasion de l'assassinat du Président Sadate deux ans plus tard, imposait de donner à la simple valeur islam une importance prioritaire, de la considérer comme moteur principal ou d'ultima ratio. La repolitisation du religieux ou l'islamisation du politique imposait presque de tout reconsidérer et d'épouser les nouvelles problématiques susceptibles d'expliquer le mouvement de bascule iranien et de comprendre l'attentat du Caire (cf. Olivier Carré, Mystique et politique: lecture révolutionnaire du Coran par Sayyid Qutb, Frère musulman radical, 1984, et Gilles Kepel, Le Prophète et Pharaon. Les mouvements islamistes dans l'Égypte contemporaine, 1984), ou la «montée » des «courants islamistes ", le «renouveau » de l'islam (cf. Shireen Hunter, éd., The politics of islamic revivalism, 1988) en terre musulmane et plus particulièrement au Maghreb (cf. Bruno Etienne, L'islamisme radical, 1987 ; François Burgat, L'islamisme au Maghreb : la voix du Sud, 1988). 
7 On se situait à l'extrême opposé de l'ouvrage de Daniel Lerner - The passing of traditional society: Modernizing the Middle East, un des premiers essais de spécialiste sur le MoyenOrient paru il y a une trentaine d'années (1958). Et l'on assistait à une floraison d'ouvrages, qui se chiffrent par dizaines sur les rapports entre "islam et politique", " islam et société ", « islam et relations internationales » dont il serait vain de dresser l'inventaire, et dont certains sont de grande qualité à l'image des essais présentés par Adeed Dawisha (Islam in foreign policy, 1985). De l'ouvrage de James P. Piscatori (Islam in a world of Nations-States, 1986), ou, pour quitter exceptionnellement le monde arabe, celui qu'Olivier Roy a consacré à l'Afghanistan: Islam et modernité politique (1985). A l'occasion, quelques grands islamologues en profitent pour rappeler aux jeunes générations qu'elles ne font que redécouvrir le monde, comme le leur ont signalé Bernard Lewis (The political language of islam, 1988), Ira Lapidus (in Edmund Burke et Ira Lapidus, éd., Islam, politics and social movments, 1988), Mohammed Arkoun et Maxime Rodinson, entre autres...

8 En un sens la part déterminante du religieux dans la mécanique politique redonnait au monde arabe une spécificité que la politologie orthodoxe - d'obédience nordaméricaine, qui n'a cessé de prévaloir en Occident depuis les années 1950 - tendait à lui dénier au nom de la double universalité des objets et des disciplines. Et, peu à peu, venait à se dégager l'idée qu'après tout les pays, les États, les nations; les communautés politiques arabes, avaient en elles - de par leurs origines, leurs histoires, leurs cultures, leurs expériences, leurs modes organisationnels - de quoi pratiquer une sorte de développement autonome, comparable à d'autres cultures, expériences, etc., mais non réductibles ou assimilables à elles.

9 A ce stade, et sans retomber dans les pièges de la spécificité qui expliquerait tout «en dernière instance », on - la politologie externe à l'objet monde arabe - était prêt à accepter l'idée que les formes de la participation politique, les modes de la relation société-État, privé-public, religieux-politique, pouvaient être à la source d'un exercice démocratique non mesurable à l'aune de ce qui s'était construit dans les milieux scientifiques et académiques - mais aussi dans des institutions moins ouvertement désintéressées (Rand Corporation, Brookings Institution aux USA, IFRI en France) nord américains et européens.

Les paradigmes de substitution

10 La démocratisation en pays arabe, les politologues occidentaux n'ont cessé d'en parler depuis trente ans. Ils ont même cherché à repérer au sein de chaque société et État ce qui pouvait bien aider à sa réalisation ou à son accélération, au travers des trois « $\mathrm{D}$ " combinés par H. Maddick (Democracy, Decentralization and Development) dès 1963. D'abord préoccupés des actions des gouvernants, susceptibles de faire avancer les processus de participation à la vie politique, de représentation populaire, ils se sont attachés aux capacités de mobilisation des régimes. Par le biais, notamment, de partis politiques monopolistes ou dominants ; qu'on se souvienne de l'essai de Clément Henry Moore sur la Tunisie, qui date de 1965 (Tunisia since independence). Ou par l'intermédiaire des régimes prétoriens présentés comme modernisateurs de type turc, égyptien ou algérien, ce qui a fait l'objet d'une littérature plus qu'abondante aux États-Unis et de plus rares essais en France (B. Vernier, Armée et politique au Moyen-Orient, 1966) ou encore par le biais des élites politiques de la nouvelle génération, celle de l'aprèscolonisation, comme en témoigne l'ouvrage de William Quandt, publié en 1963 (Revolution and Political leadership : Algeria). 
11 Ensuite, on s'est penché sur les intermédiaires sociaux de la démocratisation, non plus la « nouvelle classe moyenne » chère à Manfred Halpern (The politics of social change in the Middle East and North Africa, 1963), ou à ses équivalents mais les groupes de la socialisation politique: familles, clans, groupes ethniques pour les amateurs de la retraditionalisation et aussi pour les sociologues de la parenté et de l'honneur, tel Pierre Bourdieu (Esquisse d'une théorie de la pratique, 1972), les sociologues de la culture (Shmuel Eisenstadt) et les anthropologues du socio-culturel, tel Clifford GEERTZ (in Clifford Geertz, Hildred Geertz et Laurence Rosen, Meaning and order in Moroccan society, 1979). On s'est attaché aux syndicats, associations, guildes... à quoi il faut ajouter écoles militaires, bureaucraties, universités, pour les modernistes ; aux recettes économiques du progrès : industrialisation, et capitalisation par les États, augmentation du niveau de vie, plus tard privatisation, pour les développementalistes.

12 On inscrira sur cette trajectoire une réflexion nous ramenant aux leaders politiques et qui prenait en compte les visions, perceptions et prospectives politiques des chefs d'État sur le modèle nassérien de l'Idéologie de la révolution. Réflexion qui a permis de cerner et définir les idéaux démocratiques d'acteurs primordiaux dont Michel Camau a donné une vue d'ensemble pour ce qui est de l'Afrique du Nord dans La notion de démocratie dans la pensée des dirigeants maghrébins (1974), et que l'on trouve disséminée dans un grand nombre d'ouvrages traitant de tel ou tel pays en particulier. Avec, comme d'habitude, des distorsions aboutissant à attribuer à la personnalité d'un individu, le leader plus ou moins charismatique, des traits et orientations propres à un régime, une communauté comme à «idéologiser» et personnaliser à la fois des méthodes de gouvernement, sous le non de nassérisme, bourguibisme, kadhafisme...

Récemment on s'est tourné vers l'observation de ce que l'on pourrait appeler les ensembles sociaux, en cherchant à repérer en leur sein tantôt les formes traditionnelles capables de faire l'objet d'une redynamisation, tantôt les forces d'adaptation susceptibles de favoriser, sinon de promouvoir, des systèmes politiques de caractère plus égalitaire. Sans cesser de s'interroger pour autant sur les types et formes de régimes en activité, sur les «centralismes » et "personnalismes » politiques liés aux leaderships populaires ou autocratiques, les monarchies héréditaires ou non, les républiques libérales ou autoritaires, appuyés par (et sur) des partis uniques ou multiples, ou plutôt sur des clientèles, coteries, familles élargies ou clans. Et nous avons tout l'éventail des études sur les systèmes politiques observés à partir des chefs d'État (amîr, za'îm, ra'îs...), des souverains se réclamant de la légitimité musulmane (commandeur des croyants marocain, roi d'Arabie Saoudite, émirs du Golfe, sultan d'Oman), des pouvoirs patrimoniaux ou néo-patrimoniaux. Les ouvrages publiés au cours des années 1970 et cherchant des explications à partir des « centres » ou sommets ne manquent pas ; impossible de les citer ici.

On s'est aussi préoccupé de saisir ce qui pouvait assurer une stabilité politique, permettre de résister aux mutations sociales et qui ne s'effectuait pas aux dépens des entreprises de démocratisation (cf. Jean Leca : «Social structures and political stability. Comparative evidence from Algeria, Syria and Irak», in Adeed Dawisha et I. William Zartman, éd., Beyond coercion : the durability of the Arab State, 1988). On a poussé l'analyse en direction des politiques économiques et de leurs impacts sur les structures sociales et les relations politiques, à l'image de ce qu'ont tenté Michel Chatelus et Yves Schemeil (Les politiques industrielles et les problèmes des politiques industrielles dans les pays arabes, 1986), William Zartman (The political economy of Morocco, 1986) et tout récemment Alan 
Richards et John Waterbury (A political economy of the Middle East. State, class and economic development, 1990).

Mais le plus souvent, la démocratisation dont il pouvait être question, entre les lignes mais quelquefois de la façon la plus explicite et la plus brutale, c'était en premier lieu une modernisation construite sur une référence universelle.

C'était aussi la reproduction - ou le rapprochement en direction - du modèle ou de l'idéal-type construit à partir des processus historiques occidentaux. Ce qui servait de repère mais aussi de but, il faudrait presque dire d'utopie, c'était donc une démocratie libérale avec ses codes et normes, un type de participation politique, des libertés publiques d'un certain genre, un équilibre entre demandes sociales et redistribution économique, l'établissement d'un système égalitaire caractérisé... Ceci venait, en lignes brisées, des grands ancêtres bien sûr, et médiatisé par des pensées aussi diverses que celles de Carl Friedrich (Constitutional government and politics, 1937). Joseph Schumpeter (Capitalisme, socialisme et démocratie, 1942), Barrington Moore (Social origins of dictatorship and democracy, 1966) John Plamenatz (Democracy and illusion, 1973) pour ne retenir que quatre auteurs. Renvoyons à des essais, tout aussi dissemblables de M. I. Findley (Democracy, ancient and modern, 1973), Tatu Vahanen (The emergence of democracy : a comparative study of 119 States, 1850-1979, 1984) mais aussi de J. R. Pennock et J. W. Chapman, éd. (Liberal democracy, 1984) ou Kenneth Mc Rae (Consociational democracy: Political accomodation in segmented societies, 1974) dont la lecture est conseillée aux étudiants US en "governmen». Un ouvrage récent de Barry Holden (Understanding liberal democracy, 1988) donne une idée aussi floue du concept que forgée à partir de l'exemple historique des États-Unis. Or l'idéalisme américain des années 1960 - ce sur quoi revient Robert Dahl dans Democracy and its critics (1989) - n'est pas tout à fait innocent en ce domaine et qu'on en perçoive la trace ou les retombées aujourd'hui encore n'étonnera pas. Par contre, il est plutôt déconcertant de constater qu'une certaine sociologie politique française a épousé ce libéralisme-là, par imitation, au lieu d'emprunter ses modèles à l'héritage national construit à partir des perspectives d'ailleurs non réductibles à un même "démocratisme " de Montesquieu, Rousseau, Voltaire, Tocqueville...

17 Même si la fin des théories de la modernisation et la mise en sommeil des paradigmes fonctionnalistes ont conduit à ne plus se référer à la " grande » tradition occidentale et à reconnaitre des "petites " traditions produites par les cultures locales, ce qu'on pourrait nommer le référent imaginaire central demeurait d'essence étrangère aux milieux, restait exogène, suprême en quelque sorte.

La notion - je n'ose pas dire le concept - de mobilisation (la capacité de réunir et de faire bouger ensemble, dans une direction donnée, une communauté socio-politique et dont Ilya Harik a fait une brillante application dans The political mobilization of peasants : a study of the Egyptian community, 1974) n'est restée là souvent qu'alourdie de présupposés propres à l'Occident, liés à ce que celui-ci imaginait que la démocratie devait être. Alors, lorsque les dynamiques de rassemblement, les institutions d'encadrement, de regroupement des intérêts, de canalisation des tendances, longtemps prônés par une intelligentsia de professeurs et chercheurs prêts à imposer leur doxa au monde, aboutissaient non à des régimes représentatifs égalitaires mais à des bureaucraties omnipotentes, à des États-forteresses (du type Irak ou Syrie où, sous la bannière du Ba'th, exercent des groupes monopolistes), à des États rentiers ou « allocataires » selon la terminologie de Giacomo Luciani (cf. H. Beblawi et G. Luciani : 
The rentier State, 1987) applicable à des pays comme l'Arabie Saoudite, l'Irak, le Koweit, les Emirats arabes unis. Bahrein, Oman, la Libye, quelquefois gérés par des castes et monarques se présentant comme représentants de Dieu ou du Prophète sur terre, mais aussi et surtout à des gouvernements autoritaires, jetant les opposants en prison, réduisant en cendres la moitié d'une ville, pourchassant des minorités religieuses, gazant ou déportant un groupe ethnique réfractaire, transformant la participation populaire en une mascarade, alors les analystes criaient au scandale, aux effets pervers, évoquaient la déviance, pleuraient la démocratie bafouée. Des régimes «tribalodynastiques » ou « tribalo-républicains » de la péninsule arabique présentés par Bahgat Korany (coauteur de Les régimes politiques arabes, 1990) ou des "républiques socialistes", «monarchies libérales", «pluralismes non monarchiques» tels qu'évalués par Alan Richards et John Waterbury (A political economy of the Middle East...), peu échappaient à l'ostracisme. Autrement dit, peu méritaient de figurer dans la catégorie des États organisés selon les principes de la démocratie, en dépit des « limites » de celles-ci (cf. Crawford B. Mac Pherson, Principes et limites de la démocratie libérale, 1985).

Et nous, les experts du dehors, nous nous sommes demandés quels virus avaient touché des sociétés pour les faire sortir de la ligne les menant, même au prix de quelques méandres, à se conformer à la démocratie selon les canons définis à partir d'une pratique athénienne légendaire, telle que la rappelle R. K. Sinclair dans un fort bel essai (Democracy and participation in Athens, 1988).

Jusqu'à ces derniers temps, une certaine ambiguïté pouvait encore planer sur cette référence absolue. Parce que le marxisme avait enfanté un contre-modèle, une contreculture, des contre-institutions, et Marx lui-même puis ses épigones avaient longuement expliqué que la démocratie libérale n'était qu'une illusion, une poudre aux yeux que la bourgeoisie toute puissante avait lancée à la tête des membres des autres classes sociales, depuis que la fiction dénommée souveraineté nationale née de la Révolution française avait abouti à l'éradication de la vraie souveraineté, populaire celle-là, faisant directement appel aux citoyens, non à leurs représentants. Nous avions donc, avec le vocable de démocratie populaire, un contre-type (cliché négatif inversé et non copie) un peu gênant; en tout cas susceptible de venir à tout moment se mettre en travers des plus belles démonstrations. L'effondrement de l'Union soviétique, l'échappée des communautés politiques sous sa dépendance en Europe orientale et la conversion proclamée de tout l'ensemble à l'économie du marché, à quoi se sont ajoutés la stagnation et l'échec d'expériences américaines ou africaines (Cuba, CongoBrazzaville. Ethiopie, Angola, Mozambique) mais aussi arabes (Yémen du Sud. RDPY), tout ceci à conduit à couvrir soudain d'opprobre une autre voie démocratique possible puis à la jeter aux orties. Et en même temps on balançait par-dessus bord les théories de la dépendance qui auraient pu survivre. La démocratie à l'occidentale pouvait donc triompher! Même si quelques défenseurs des orthodoxes agonisants tentaient de constituer un dernier carré, à l'image de l'équipe rassemblée par Peter Anyang Nyongo et patronnée par Samir Amin (Afrique : La longue marche vers la démocratie. État autoritaire et résistances populaires, 1988) que l'on pourra comparer avec une série d'essais moins systématiques en langue anglaise publiés la même année (Democracy theory and practice in Africa) ou à l'ouvrage dirigé par Larry Diamond, Juan Linz et Seymour Martin Lipset (Democracy in developing countries, Vol. 2, Africa).

Les modèles récusés 
21 Commençons par dire que les seules « démocraties » du Moyen-Orient, du point de vue d'une certaine politologie, française et anglo-saxonne, nommément le Liban et Israël, et qui auraient pu faire naître des modèles autochtones, une société politique fondée sur le pluriconfessionalisme ici et sur l'idéologie du kibboutz - autrement dit sur l'exploitation communautaire - là, ces deux démocraties se sont effondrées.

22 La première, cette "Suisse du Moyen-Orient » comme l'enseignait au début des années 1960 un des grands maîtres de la science politique française, Maurice Duverger et qu'évoquaient Pierre Rondot (Les institutions politiques du Liban, 1947) et Chartes Rizk (Le régime politique libanais, 1966) mais que Michael Hudson qualifiait de Precarious Republic (Modernization in Lebanon), dans un ouvrage daté de 1963 et augmenté en 1985, ce que confirmait l'essai de Georges Corm sur le pluralisme multiconfessionnel (Contribution à l'étude des sociétés multiconfessionnelles) paru trois ans plus tard, le Liban a éclaté, faute d'avoir transcrit dans la sphère politique des représentations et de la gestion de l'État les modifications apportées dans les rapports de poids entre les communautés religieuses, ne serait-ce que du seul fait de la démographie, d'avoir rompu aussi les pouvoirs des caciques ou de ceux qui, se substituant aux vieux chefs, ont continué à jouer des haines fratricides selon les termes d'Elisabeth Picard (Liban, État de discorde: des fondations aux guerres fratricides, 1988).

La seconde s'est pervertie : en Israël, l'idéologie communautaire appartient désormais au mythe fondateur. Par contre, le parlementarisme n'a pu produire que le contraire de ce à quoi il devait aboutir, à l'influence hors de proportion des mini-partis sur les décisions et des extrémismes sur les actions, sans parler des méthodes employées pour maintenir « l'ordre » (israélien) dans les « territoires occupés » (Palestine).

D'autres États auraient pu servir de modèles, à tout le moins fournir des maquettes où l'Occident aurait pu se reconnaître. La Libye du Livre vert a fait illusion un temps. Et quelques commentateurs, issus des milieux occidentaux de la recherche et de l'enseignement, sont allés apporter leur caution lors des grandes cérémonies destinées à célébrer la voie libyenne vers la démocratie directe. Des livres ont vanté les comités populaires, l'égalitarisme en mouvement, le pouvoir partagé, le leader charismatique non autoritaire... D'autres sont allés plus profond et ont démonté les mécanismes d'un gouvernement et retrouvé les origines d'un pouvoir, tel John Davis (Lybian politics : tribe and révolution, 1988) conduisant à une démythification et à une meilleure appréciation du réel. Ce qui n'a pas empêché le général Umar al-Bachir, le chef de la junte de Khartoum de proposer l'adoption, pour son pays, du système des comités populaires copié sur la Jamahiriyya libyenne, à l'issue de la "conférence nationale de dialogue », énième mouture destinée à résoudre la quadrature du cercle ethnico-religieux soudanais.

Un moment, ce même Soudan a aussi présenté un caractère exemplaire. Très précisément lorsque l'Armée s'est retirée et a cédé le pouvoir aux civils après un large soulèvement populaire. Le retour de Sadiq al-Mahdi à la direction des affaires politiques après vingt ans de mise à l'écart - à la suite d'élections libres et de la sélection d'une assemblée constituante au suffrage universel (avril 1986) et après autant d'années ou presque de régime militaire - représentait le retour de (et à) la démocratie, du multipartisme, de l'expression naturelle de forces issues de la vie confrérique propre à l'islam soudanais (Ansar, Khatimiyya) et des regroupements politiques (Parti communiste, Frères musulmans). Ainsi se trouvaient rétabli dans ses droits un régime pluraliste né dès avant la fin du condominium franco-britannique, 
libérée une opinion, réaffirmé un mode de gouvernement, étaient transcris des choix. Les élections de 1986 réintégraient donc le Soudan dans une tradition libérale fort bien reconstituée par Tim Niblock dans Class and power in Sudan. The dynamics of Sudanese politics 1898-1985 (1987), donc dans le « camp » des démocraties, selon tous les analystes d'alors (cf. Revue de la Presse égyptienne $\mathrm{n}^{\circ}$ 22, 1-1986). Depuis, la guerre civile et l'autonomisme du Sud n'ont pu être résorbés au prix de quelque compromis et la situation économique s'est dégradée. Les renversements d'alliances politiques ont rompu l'équilibre précaire au sein du gouvernement, la voie était libre pour un système militaro-islamique, le premier du genre. L'Armée s'est en effet à nouveau emparée du pouvoir : un général rechaussant les bottes laissées tièdes par Nimeiry. Et l'on assiste à une faillite de l'ensemble de l'élite politique telle que la proclame un Mansour Khalid (rallié aux Sudistes) dans son The government they deserve. The role of the elite in the Sudan's political evolution (1990). Aujourd'hui, les opposants sont pourchassés, les libertés suspendues, les partis interdits... le tout au nom de l'islam.

Quelques observateurs ont voulu saluer le changement de régime du Yémen du Nord marqué par la chute de l'imamat et l'instauration concomitante d'une république socialiste, en 1962. L'intervention des militaires marquait non pas l'installation d'un régime prétorien mais la fin d'un système théocratique, donc "libérait» une communauté politique, en tout cas en apparence. A y regarder de plus près, les mêmes ont dû déchanter et reconnaître que les mécanismes politiques n'avaient conduit à aucune démocratisation effective et que la société yéménite n'était pas modifiée. D'autres ont suggéré que la monarchie parlementaire marocaine représentait une équation canonique et répondait à un certain nombre de réquisits. Ceci dans le droit fil de ce que suggérait, dès l'indépendance acquise, Jacques Robert dans La Monarchie marocaine et que tentèrent de démontrer plus de vingt ans après Georges Vedel et les auteurs de Édification d'un État moderne. Le Maroc de Hassan // (1986). Entre-temps, les thèses de John Waterbury (The Commander of the faithful. The Moroccan political elite: a study in segmented politics, 1970) et de Rémy Leveau (Le fellah marocain défenseur du trône, 1976 et 1985) avaient, entre autres études, éclairé sur les dérivations du régime. L'arbitraire du monarque, la domestication des partis, ont depuis lors conduit les inconditionnels à revenir sur leur premier jugement.

L'ultime secteur de projection d'une démocratie à créer, à inscrire dans une perspective nationale et un cadre étatique demeurait la Palestine, en tant que lieu de l'encore possible. Les textes de 1964-1970 présentés par Olivier Carré puis ceux, récents, rassemblés par Jean-François Legrain (1991) donnent une idée de l'idéologie palestinienne de résistance, pour reprendre le titre de l'ouvrage du premier nommé. Le livre d'Alain Gresh (OLP. Histoire et stratégie: vers l'État palestinien, 1983) fournit une possible approche de ce que pourrait être l'appareil indépendant de gestion d'une communauté politique rendue à elle-même. Les occidentaux peuvent y reporter leurs espoirs lorsqu'ils ne sont pas perturbés par l'omniprésence d'Israël, ce qui n'est pas le privilège de chercheurs américains, tant s'en faut.

Restait l'islam justement, comme porteur possible d'une formule politique originale de type démocratique. A ceci près que si on s'impose la lecture de la masse d'ouvrages publiés depuis une décennie sur islam et politique - pour l'essentiel outre-Atlantique mais aussi en Europe, et écrits par des gens qui, quelques années auparavant, ne s'en souciaient nullement, donc sous l'effet iranien répétons-le - on ne trouve guère d'ébauche d'un modèle original. 
29 Mieux, ou pire, les politologues à l'œuvre - lorsqu'ils ont retrouvé des modèles possibles d'une démocratie islamique grâce à la consultation des grands juristes, jurisconsultes, penseurs, philosophes politiques, musulmans et/ou à la lecture des islamologues européens - ont vite conclu que la "Cité musulmane », dont Louis Gardet avait pourtant démontré la vitalité et les extraordinaires capacités d'adaptation, était à l'image de celles de Thomas More et de Campanella, une utopie. Quant à vouloir recréer la communauté mohammédienne des débuts de l'islam, fondée sur une base égalitaire, il n'y fallait point penser ; à quatorze siècles de distance c'était tout à fait irréaliste, aux dires des experts occidentaux.

Et puis - et le grand Samuel Huntington le dit sans détour dans l'article cité au tout début de cette introduction («Will more countries become democratic?») - « Islam has not been hospitable to democracy ». Autrement dit, en matière de démocratie, l'islam ne peut servir de source où s'alimenter. Ce que paraissaient déjà annoncer James Bill et Carl Leiden (dans Politics in the Middle East. paru en 1984, la même année que le texte de Huntington précité) lorsqu'ils affirmaient que l'histoire sociale du Moyen-Orient tendait à prouver que les formes associatives politiques étaient l'exception, et les groupes informels la règle. Et comme l'on savait par ailleurs, depuis que von Grunebaum l'avait si bien démontré, que la perception historique ou l'historicité ne participait pas de la culture orientale en général et de la culture arabe en particulier, ce à quoi venait s'ajouter l'absence de séparation entre le politique et le religieux, on imaginait autant de barrières ou d'impossibilités.

31 L'islam, aujourd'hui, pour quelques politistes - qui se présentent d'autant plus volontiers comme des experts ès-sciences religieuses islamiques que les véritables spécialistes sont rares et se gardent de se prononcer - serait même carrément contraire à la démocratie. La démonstration ne s'encombre pas de nuances ni ne tient compte de la diversité des régimes arabes en activité depuis l'époque du Prophète ni de systèmes actuels fonctionnant selon des critères à tout prendre très laïcs. Elle s'appuie sur des données simples et s'enclenche de façon syllogistique. Simplifions, au risque de paraitre caricatural :

- $1^{\circ}$ la démocratie c'est le pouvoir aux hommes, aux citoyens, qui l'exercent par la voie de représentants élus. Dans la théocratie, à l'inverse, le pouvoir vient de Dieu, le droit est divin et transite par son mandataire terrestre. Dans le premier cas l'individu, la liberté, sont des facteurs clés, les fondements. Dans le second, c'est le souverain, shérif, aidé des avis des commentateurs du Coran, qui est la base.

- $2^{\circ}$ la démocratie c'est le produit de mutations économiques, sociales et culturelles liées à la révolution industrielle en Europe et en Amérique du Nord, et qui sont l'œuvre des hommes, de leurs entreprises conjuguées. Dans le monde arabe, aucune transformation de cette intensité ne s'est produite dans les profondeurs de l'infrastructure. Et ceux qui en prennent conscience y voient l'effet même de la volonté divine, la marque d'un destin auquel se plier.

- $3^{\circ}$ les deux « modèles », comportements, mentalités, cultures politiques, sont incompatibles.

Tout ceci ne pouvait à l'évidence - toujours du point de vue des analystes étrangers à la région comme à la religion - favoriser une agrégation des intérêts, une prise de conscience politique, autrement dit la formation des esprits et la représentation de type moderne. En un mot, la tradition islamique n'apparaissait pas porteuse des " gènes politiques » propres à la naissance de la démocratie; plus précisément des notions d'égalité, de participation, de liberté, de contestation... A preuve ces élections plus ou moins biaisées, ces votes télécommandés, ces assemblées introuvables aux majorités 
inamovibles, en Égypte, au Maroc, ce parlement libanais prorogé depuis 1972 de législature en législature et probablement jusqu'à la mort du dernier député ! A preuve ces oppositions-fictions, ces partis dominants devenus partis-paravents, ces droits de l'homme non respectés, ces émirats et monarchies d'un autre temps, ces bureaucraties rentières, ces États banquiers, ces oligarchies pétrolières... A preuve ces militaires s'emparant du pouvoir de façon durable et imposant des procédures quasi dictatoriales pour sauver la paix sociale de sociétés qui n'en demandaient pas tant ou pour préserver l'unité nationale de nations qui se seraient volontiers contentées d'autres régimes. A preuve ces quelques mouvements extrémistes se réclamant d'un islam pur et dur et prônant une "démocratie" d'essence prétendument musulmane et profondément inégalitaire et allant jusqu'à l'assassinat d'un chef d'État et d'un président d'Assemblée (Égypte). En de trop nombreux lieux, la démocratie était en cause, en litige, menacée. Jugée, on trial (Reflections on arab and african politics) selon les termes de M. A. Mahjoub (1974), c'est-à-dire "à l'épreuve » mais peut-être aussi "en procès ", ou encore « épuisées » « à la dérive » pour reprendre la formule de Juan Linz et Alfred Stefan (The breakdown of democratic regimes, 1978.4 vol.) appliquée il est vrai à d'autres sociétés et régions du monde.

\section{Réexamens en cours}

Depuis quelques années, l'impression prévaut que l'ethnocentrisme des sciences sociales occidentales est entamé, qu'un certain pluralisme s'est installé. L'approche culturaliste dominante s'est ouverte à l'historique autonomisé, donc aux diachronies et synchronies propres à des ensembles sociaux, des civilisations, des ethnies, des régions. En témoignent les différentes contributions qui composent l'ouvrage dirigé par Ghassan Salame The foundations of the Arab State (1987). Elle tend aussi à ne plus considérer institutions et États, sur lesquels on revient - non sans raison car ceux qui annonçaient le recul des États et le retour concomitant des sociétés s'étaient un peu trop pressés - avec les mêmes visions unilatérales qu'autrefois. Le monde arabe est abordé de façon à la fois plus pragmatique et plus « prismatique » c'est-à-dire en tenant compte de la diffraction, des nuances, des situations... Les meilleures démonstrations en sont fournies par les 4 volumes rassemblés par Giacomo Luciani sur Nation, state and integration in the arab world (1987) - qui, entre les chapitres, rend à César ce qui est à César et aux arabes ce qui leur appartient en propre en matière de construction politique - ainsi que par l'ouvrage de Bertrand Badie sur Les deux États, pouvoir et société en Occident et en terre d'islam (1987), qui réintroduit le domaine arabe dans le comparatif. On portera aussi au crédit du réformisme (ou révisionnisme, comment en décider?) ambiant, l'essai que Léonard Binder - enfant terrible et souvent critiqué d'une science politique nord-américaine du monde arabe - vient de consacrer au «libéralisme islamique ». Bien que centré sur une réfutation des théories du développement d'une part, et sur une mise en doute, sinon en cause de ce qui constitue précisément son sujet (le monde arabe peut-il produire une idéologie libérale ?) d'autre part, le livre rouvre le dialogue entre les idéologies, entre les sociétés.

A titre de démonstration de cette reconversion - faut-il l'assimiler à une «amende honorable » ? - de la politologie occidentale, je prendrai les colloques, tables rondes et autres manifestations scientifiques - évoquant à peu de chose près ce qui fait l'objet du troisième colloque franco-égyptien de politologie du Caire (septembre-octobre 1990), à savoir les processus de "démocratisation dans le monde arabe » et tenus sous l'égide des grandes institutions de recherche, le Social Science Research Council américain et 
l'Association internationale de Science politique, en tête. J'évoquerai aussi les contributions d'analystes qui n'ont cessé de traiter des questions - d'interpeller dit-on aujourd'hui - qui, au cours des dix dernières années, ont attiré l'attention des spécialistes.

Ainsi Michaël Hudson, l'auteur de Arab politics. The search for legitimacy (1977), un de ceux qui ont le plus contribué à l'étude politique du monde arabe aux États-Unis, professeur de science politique à l'Université de Georgetown à Washington, a fait un séminaire à l'Université du Koweït en 1990, sur «les perspectives de démocratisation dans le monde arabe ", thème qu'il a repris lors du congrès annuel de science politique qui s'est tenu à San Francisco au cours de cette même année 1990.

Ainsi Lisa Anderson. qui vient de terminer une étude importante sur les perspectives de la démocratie dans le monde arabe, sous le titre Democratic liberalism in the Arab world rédigée à partir d'interviews du personnel dirigeant de quatre pays : Jordanie, Égypte, Tunisie et Maroc; étude centrée sur les influences internes et externes, l'organisation politique et sociale, les enjeux idéologiques, les acteurs et leur influence sur la possible libéralisation des mécanismes politiques et de la démocratisation des régimes.

Ainsi, le $\mathrm{XV}^{\mathrm{e}}$ Congrès de l'Association internationale de Science politique (IPSA pour tout le monde y compris les francophones!) qui se tiendra à Buenos-Aires en juillet 1991, prévoit-il une dizaine d'ateliers portant sur les démocratisations en cours ("Transitions vers des régimes démocratiques ", "Système électoraux des nouvelles démocraties et des pays en voie de démocratisation», "Gouvernement militaire et démocratisation dans le tiers-monde ", «Démocratisation comparée ». "Transitions à la démocratie... ", « Démocratie et solidarité ", par exemple).

Il y aurait dix autres exemples à citer. Je m'en tiens là. Ce que je veux dire, à travers eux, c'est que la politologie occidentale a pris enfin en compte des processus démocratiques produits dans le monde arabe, sans les séparer totalement de ceux en cours dans le reste du monde. Et il faudrait réfléchir, à ce propos, à ce que pourrait être une analyse en termes de politiques-mondes, comme celles menées; en termes d'économies-mondes. Sont comptabilisés désormais les expériences parlementaires et les modes d'organisation politique de pays comme l'Égypte, le Maroc, le Liban et le Koweït il y a peu encore, voire de la Jordanie et de la Tunisie par exemple. Mais sont retenus aussi des phénomènes et systèmes ne reposant pas sur des bases pluralistes d'expression, sans pour autant leur attribuer des laps antidémocratiques. Aussi revienton sur les efforts des concentrations des pouvoirs et des centralisations des appareils de la gestion étatique en matière d'économie principalement, comme facteurs d'intégration et de redistribution des ressources, en matière de formation aussi, comme moteurs d'une acculturation politique et canaux d'une promotion sociale et professionnelle par l'éducation.

On cesse donc de .juger hâtivement ces "démocratisations par en haut " telle celle donnée par feu le Président Boumediene, décidant de recréer des assemblées consultatives locales, municipales (APC) puis des assemblées régionales, des wilayâs (APW), avant d'asseoir au sommet de la hiérarchie consultative et participationnelle des instances une assemblée populaire nationale (APN). A l'heure où le Front islamique du Salut est susceptible de déposséder l'autre "Front officiel, dit de libération nationale, du contrôle de ces différents organes par la voie toute légale des élections, et que l'ancien président Ben Bella rentre avec le projet néo-péroniste proclamé et sans doute 
illusoire de reprendre le pouvoir par le canal institutionnel, il est difficile de crier qu'une certaine démocratie n'est pas au travail.

On regrette aussi, ces jours-ci (septembre 1990), que ce que l'on nommait l'option démocratique koweïtienne n'ait pas abouti. Et on revalorise rétrospectivement, la constitution présentée comme libérale dont le régime de l'émirat s'était doté en 1961, avec un parlement élu, même s'il l'était sur des bases sociales et ethniques étroites, non sans analogie avec les cités grecques d'antan. Et l'on regrette plus encore qu'en 1976 puis en 1985, des articles de la constitution aient été suspendus et que le parlement ait été dissous. Au point de glorifier la réanimation de structures traditionnelles, les diwaniyya, susceptibles de regrouper plusieurs centaines de personnes et de débattre des problèmes de l'heure, sous l'inspiration d'anciens députés, leaders naturels d'une opposition en voie de création. Les journalistes étrangers ont parlé à ce propos de bataille pour la démocratie, où instances traditionnelles et nouvelles entraient dans une exceptionnelle combinatoire. On sait ce qu'il est advenu de cette bataille, que l'invasion irakienne aura régie à sa façon et que, à l'évidence, la monarchie saoudienne n'appréciait pas.

Il y a dans toutes ces observations comme une rupture, un changement d'attitude à tout le moins, par rapport aux visions et comportements antérieurs. Pour les chercheurs occidentaux, la démocratie n'est plus une catégorie pertinente du seul fait de sa relation aux systèmes politiques sur lesquels l'Occident a prétendu fonder son ordre politique. En d'autres termes, l'État de droit ne saurait venir en ligne unique d'Athènes ou de Sparte, à travers la tradition européenne issue des révolutions, anglaise, américaine, française et de la manière dont la liaison entre morale et politique, par l'intermédiaire du droit, a pu être établie dans la tradition kantienne. Il ne saurait, non plus, se situer dans la lignée marxiste, empruntée en partie à Morgan, celle des communautés paysannes auxquelles, d'ailleurs, les démocraties populaires défuntes ne devaient rien ou presque.

Déjà en Occident, plusieurs interprétations ont fait l'objet de concurrences. Celle, née en 1789 et qui s'attachait en priorité à la protection de l'individu, dénommé citoyen, contre l'État et ses empiètements et était fondée sur les droits de l'homme, n'en recouvrait pas forcément une seconde s'attachant surtout à l'égalité des conditions sociales et plus tard à l'égalité des chances, et qui pourrait se réclamer de Tocqueville. Une troisième version, plus actuelle, bien qu'ancrée déjà chez Rousseau pourrait avoir pour fondement premier le rapport douloureux entre personnalité et liberté, loi et expression ou mieux entre légitimité du pouvoir et mouvement social, ce qu'en termes plus élaborés exprime par exemple un Claude Lefort (L'invention démocratique, les limites de la domination totalitaire, 1981).

Il me semble que cette démocratie repensée comme pouvant être multiple, nous la devons pour partie à des politologues (Guy Hermet dans Aux frontières de as démocratie) mais aussi à des philosophes, notamment à Jean Baechler dont l'ouvrage Démocraties (1985) aide effectivement à revoir le concept dans sa globalité, et sans doute de façon plus productive que le Democracies d'Arend Lijphart(1984).

En plus de 700 pages, Jean Baechler nous dit fort bien que l'histoire est pleine de régimes démocratiques - ce que je préférerais nommer "expériences" - plus qu'il paraît et que les politologues et juristes d'Occident ont prétendu en tout cas. Au point que les formes monarchiques et impériales auraient fait figure d'exception. Cette donnée impliquant la naturalité de la démocratie peut choquer les perceptions 
occidentales classiques. Elle s'appuie tout de même sur de solides démonstrations dont on ne peut que conseiller la lecture, faute de les résumer ici. Elle ouvre aussi la voie à un comparatisme d'envergure. Elle nous invite tout cas à la prendre en compte et à ne plus traiter du monde arabe comme d'une catégorie à part, frappée de tares originelles et incapable, par essence, de produire autre chose que des ersatz ou d'imiter sans pouvoir reproduire, condamnée aux nouveaux despotismes, néo-patrimonialismes, sultanismes populaires, radicalismes pétroliers, populismes autoritaires, pétrooligarchies... et autres qualificatifs à vocation classificatoire liés à autant de retards historiques.

N.B. La littérature dont je traite, on l'aura constaté, est essentiellement nordaméricaine. C'est elle qui nous conduit sinon nous gouverne. Je n'ai guère tenu compte des travaux européens autres que français, à l'exception de quelques ouvrages britanniques.

Je suis parti d'un texte récent de Lisa Anderson : «Policy making and theory building : American political science and the islamic Middle East », chapitre d'un ouvrage publié sous la direction d'Hisham Sharabi (Contemporary scholarship of the islamic Middle East, 1990) auquel j'ai emprunté plusieurs arguments. J'ai repris aussi des réflexions personnelles et des éléments rassemblés en vue de la préparation de diverses rencontres et conférences, portant plutôt sur le Maghreb.

Je renvoie aux essais, aussi riches que vigoureux et systématiques de Jean Leca: "L'économie contre la culture dans l'explication des dynamiques politiques" in Récentes transformations politiques dans le monde arabe, CEDEJ, le Caire. 1988 et "Globalisation / universalité en science politique, source, manifestations, résultats " contribution au colloque ayant pour thème L'étude $d u$ monde arabe contemporain. Approches globales et approches spécifiques (3-5 juillet 1989) dont les Actes doivent paraître courant 1991 (CEDEJ. le Caire), ainsi qu'aux autres textes préparés pour cette rencontre. La contribution présentée par Michel Camau « Trois questions à propos de la démocratisation dans le monde arabe", à l'occasion de la $3^{\text {e }}$ rencontre francoégyptienne de politologie Démocratie et démocratisation dans le monde arabe (Le Caire, 29-30 septembre-1er octobre 1990), approfondit quelques points que je n'ai fais qu'évoquer. On se reportera aussi à la version multigraphiée du texte de Ghassan Salame, "Problématique de la démocratie dans le monde arabe", préparé pour la session inaugurale du Comité culturel consultatif de l'Institut du monde arabe (janvier 1990). Enfin, je signale l'intérêt que peut présenter, pour une nouvelle lecture des systèmes politiques arabes, la seconde édition des Régimes politiques du monde arabe (1990) et la lecture du court et dense article de Ali Eddîne Hilal Dessouki, paru dans la revue Monde arabe, Maghreb Machrek ( $\mathrm{n}^{\circ} 127,1^{\mathrm{er}}$ trimestre 1990, pp. 6-16) sous le titre «L'évolution politique de l'Égypte : pluralisme démocratique ou néo-autoritarisme ». 


\section{NOTES}

1. La présente contribution a introduit le colloque « Démocratie et démocratisation dans le monde arabe » organisé par le CEDEJ et le Centre d'études et de recherches politiques de l'Université du Caire (voir texte de présentation).

\section{AUTEUR}

JEAN-CLAUDE VATIN

CEDEJ-CNRS 